\title{
Process Optimization of MTBE Reactive Distillation Using GSA
}

\author{
Vandana Sakhre, Sanjeev Jain, V. S. Sapkal, and D. P. Agarwal
}

\begin{abstract}
In this paper we have used the heuristic search algorithm for the process optimization of Reactive Distillation column. Basically, Process optimization is the manipulation of process variables, so as to optimize some of the parameters without violating the constraints. Gravitational Search Algorithm (GSA) is a new heuristic optimization technique based on law of gravity and mass interactions. This technique is used for process optimization of Methyl-Tert-Butyl-Ether (MTBE) reactive distillation. This work highlights the potential of GSA for an optimization of MTBE reactive distillation that involves complex reaction system. The results obtained gives better performance of MTBE reactive distillation.
\end{abstract}

Index Terms-GSA, heuristic algorithm, MTBE, optimization.

\section{INTRODUCTION}

In a process industry, Reactive distillation (RD) combines both reaction and separation operation in a single column. This technique is especially useful for equilibrium-limited reactions such as Esterification, Ester hydrolysis, Etherification and Polymerization reactions. By using RD conversion can be increased due to the continuous removal of reaction products from the reactive zone. This has a great impact on by reducing capital and investment costs and help in growth and development due to a lower consumption of resources [1]. The use of Reactive Distillation for a particular application depends on various factors such as volatilities of reactants and products along with the feasible reaction and distillation temperature.

The whole reactive column divided into three sections rectifying, reactive and stripping zones which leads to complex interactions between vapor and liquid equilibrium, mass transfer rates, diffusion and chemical kinetics, but it leads to a great challenge for design and synthesis issues [2] In the reactive column the conditions present are suboptimal both for chemical reaction and simultaneous separation. In a liquid-phase reaction systems, Reactive distillation play a very useful role as the reaction must be carried out with a large excess of one or more of the reactants. The basic etherification reaction between mixed isobutene with methanol to produce Methyl-Tert-Butyl-Ether (MTBE) in the presence of a strong acid catalyst [3].

The global optimizations of chemical processes using stochastic methods are generally used in process

Manuscript received February 9, 2014; revised May 6, 2014.

Vandana Sakhre and Sanjeev Jain are with Madhav Institute of Technology \& Science, Gwalior, India (e-mail: vssakhre@gmail.com).

V. S. Sapkal is with RTM Nagpur University, Nagpur, India.

D. P. Agarwal is with UPSC, New Delhi, India. optimization. Stochastic methods have capacity to escape local optima and find solutions in the vicinity of the global optimum. In research carried out by Edwin Zondervan [4], [5] they have emphasized on Mixed Integer Non Linear Programming (MINLP) model to get optimized design of reactive distillation column. MINLP is combined with General Algebraic modeling System (GAMS), and it can be used to solve locally as well as globally. The other method given is Mixed integer dynamic optimization (MIDO) is used when both discrete and continuous decision are to be made in process plant. MIDO is suited for practical solution of large engineering problems [6].

Gravitational Search Algorithms (GSA) is heuristic search algorithm based on universal gravity and mass interaction. GSA, developed in the past few years, as a flexible and well balanced strategy to improve exploration and exploitation methods and the binary gravitational search algorithm was developed to solve different nonlinear problem [7]. An early work of the authors has successfully adapted this technique to the cell placement problem, and shown its efficiency in producing high quality solutions in reasonable time. It needs refinements, as in many other nature inspired algorithms, to maximize its performance in solving various types of problems. Gravitational Search Algorithms (GSAs) are novel heuristic optimization algorithms and developed in the past few years, as a flexible and well balanced strategy to improve exploration and exploitation methods and the binary gravitational search algorithm was developed to solve different nonlinear problem [8]. To assess its performance and robustness, they compare it with that of Genetic Algorithms (GA), using the standard cell placement problem as benchmark to evaluate the solution quality, and a set of artificial instances to evaluate the capability and possibility of finding an optimal solution. The results show that the proposed GSA approach is competitive in terms of success rate or likelihood of optimality and solution quality [9].

In our paper we have assessed the performance of GSA and its robustness, for MTBE reactive distillation system. The results show that the proposed GSA approach is competitive in terms of success rate or likelihood of optimality and solution quality.

The organization of this paper is as follows:

Section II includes process model of MTBE reactive distillation. Section III describes process optimization of MTBE reactive distillation using GSA. Section IV includes results and discussion and Section $\mathrm{V}$ gives conclusion.

\section{PRocess Model of MTBE ReACtive Distillation}

In the last two decades, research on heterogeneously 
catalyzed reactive distillation has been extended to various industrial processes, including a well-known process for the production of octane booster Methyl-Tert-Butyl-Ether (MTBE), methyl acetate process and Nylon 6,6 process. The Schematic Diagram of Reactive Distillation is shown in Fig. 1. Most of reactive distillation mathematical models are originally derived from conventional distillation calculations, which are based on equilibrium stage model. The liquid and vapor phase are assumed to be in phase equilibrium, and the stage is associated with the MESH equations (Material balance, Equilibrium relationship, Summation (constraint) and Heat (energy) balance. Relaxation method can be used as a solving tool for reactive distillation model with the MESH equations written in unsteady state form and thus utilizing numerical integration to find the steady-state solution [10].

The overall material balance for equilibrium stage $j$ :

$$
F_{j}+L_{j-1}+V_{j+1}+\delta_{j} R=L_{j}+V_{j}(\mathrm{~mol} / \mathrm{s})
$$

Unsteady state component i material balance:

$$
\begin{aligned}
H_{j} d \frac{x_{i}}{d t}=\left(z_{j, i} F_{j}\right. & \left.+x_{j-1, i} L_{j-1}+y_{j+1, i} V_{j+1}\right) \\
& -\left(x_{j, i} L_{j}+y_{j, i} V_{j}\right) \\
& +\delta_{j} \sum\left(v_{r, i} R_{j, i}\right)(\mathrm{mol} / \mathrm{s})
\end{aligned}
$$

Energy Balance:

$$
\begin{aligned}
\dot{m}_{j} \dot{H}_{j}^{L}=L_{j+1} H_{j+1}^{L} & +V_{j-1} H_{j-1}^{V}+F_{j} H_{j}^{F}-\left(L_{j}+S_{j}^{L}\right) H_{j}^{L} \\
& -\left(V_{j}+S_{j}^{V}\right) H_{j}^{V} \\
& +R_{f} \sum_{j=1}^{N_{r}}\left(r_{j, n} \varepsilon_{j} \Delta H_{r, j, i}\right)-Q_{j}
\end{aligned}
$$

Equilibrium:

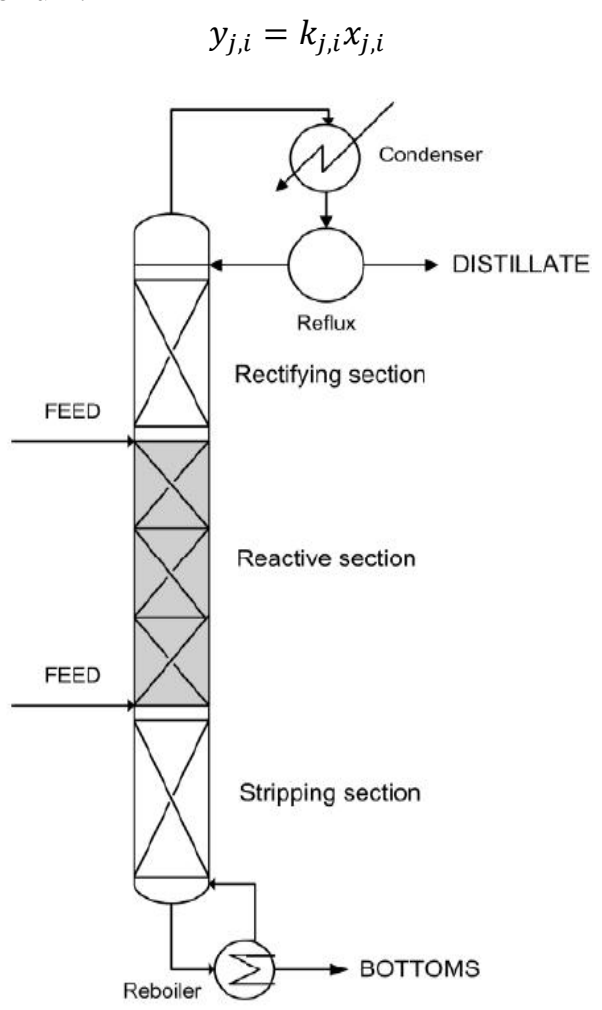

Fig. 1. Schematic diagram of reactive distillation.

\section{Process Optimization OF MTBE REACtive DISTILLATION BY GSA}

In chemical engineering process design or plant operation we can have many solutions. Hence, Optimization plays an important role in selecting the best among the entire set using some of the optimizing methods including heuristic methods.

The better optimization results can be obtained by critical analysis of process variables and detailed process design. The selection of appropriate process variable as performance objective with key parameters which affects the process is important decision variable to get optimal solution. It is required to systematically identify the objective, process constraints, and degrees of freedom in a process or a plant to improve the quality of design and faster decision making [11]. In [12], the author implemented range of nonlinear function optimization using particle swarm optimization technique with neural network training. In [13], the author used hybrid approach of Genetic Algorithm (GA) and Bacterial Foraging (BF) techniques for function optimization. In [14], the author implemented constrained optimization Augmented Lagrangian Particle Swarm Optimization (ALPSO), for tuning PID controller. To gain maximum value of objective function the metaheuristic algorithm like Central Force Optimization (CFO), is used, which is a new deterministic multi-dimensional search based on the metaphor of gravitational kinematics [15].

The GSA starts with a set of agents, selected at random with certain positions and masses representing possible solutions to a problem, and iterates by changing the positions based on some values like fitness function, velocity and acceleration. The sequential Flow Chart of GSA Algorithm is shown in Fig. 2.

In this research study, we have used the general equations proposed by Rashedi [7].

In a system with $\mathrm{N}$ agents (masses), we define the position of the $i^{\text {th }}$ agent by:

$$
X_{i}=\left(X_{i}^{1} \ldots . X_{i}^{d} \ldots . X_{i}^{H}\right) \text { for } i=1,2, \ldots . N
$$

where $x_{i}^{d}$ present the position of the $i^{\text {th }}$ agent in the $d^{\text {th }}$ dimension.

At a specific time $t$ the force acting on mass $i$ from mass $j$ is presented as follows:

$$
F_{i j}^{d}(t)=G(t) \frac{M_{p i}(t) \times M_{a j}(t)}{R_{i j}(t)+\varepsilon}\left(x_{j}^{d}(t)-x_{i}^{d}(t)\right)
$$

where $M_{a j}$ is the active gravitational mass of agent $j, M_{p i}$ is the passive gravitational mass of agent $i, G(t)$ is gravitational constant at time $t, \varepsilon$ is a small constant, and $R_{i j}(t)$ is the Euclidian distance between two agents $i$ and $j$ :

$$
R_{i j}(t)=\left\|X_{i}(t), X_{j}(t)\right\|_{2}
$$

The total force acting on mass $i$ in the $d^{\text {th }}$ dimension in time $t$ is given as follows:

$$
F_{i}^{d}(t)=\sum_{j=i j \neq i}^{N} \operatorname{rand}_{i} F_{i j}^{d}(t)
$$

where $r$ and $i$ is a random number in the interval $(0,1)$. The acceleration related to mass $i$ in time $t$ in the $d^{\text {th }}$ dimension is given as follows: 


$$
a_{i}^{d}(t)=\frac{F_{i}^{d}(t)}{M_{i i}(t)}
$$

where $M_{i t}$ is the inertial mass of $i^{\text {th }}$ agent.

Gravitational constant, $G$, is initialized at the beginning of the search and will be reduced with time to control the search accuracy as follows:

$$
G(t)=G\left(G_{0,}, t\right)
$$

where $T$ is the number of iteration, $G_{0}$ is given constant.

Gravitational and inertia masses are simply calculated by the fitness evaluation. Updating the gravitational and inertial masses by the following equations:

$$
\begin{gathered}
M_{a i}=M_{p i}=M_{j i}=M_{i}, \quad i=1,2, \ldots \ldots N \\
m_{i}(t)=\frac{f i t_{i}(t)-\operatorname{worst}(t)}{\operatorname{best}(t)-\operatorname{worst}(t)}, \\
M_{i}(t)=\frac{m_{i}(t)}{\sum_{j-1}^{N} m_{j}(t)},
\end{gathered}
$$

where $f i t_{i}(t)$ represent the fitness value of the agent $i$ at time $t$, and, $\operatorname{worst}(t)$ and best $(t)$ are given as follows for a minimization problem:

$$
\begin{aligned}
\operatorname{best}(t) & =\min _{j e(1 \ldots N)} f i t_{j}(t) \\
\operatorname{worst}(t) & =\max _{j e(1 \ldots N)} f i t_{j}(t)
\end{aligned}
$$

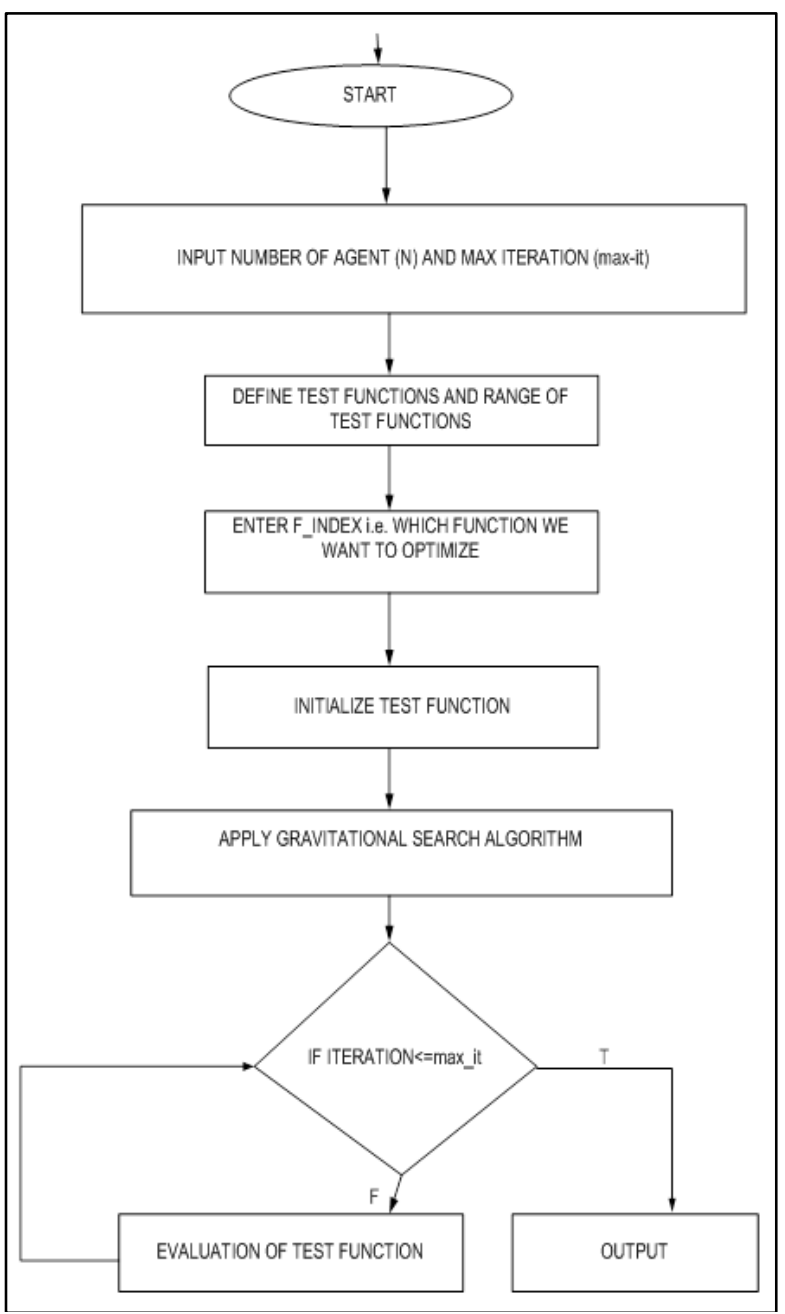

Fig. 2. Flow chart of GSA algorithm.
In our case of MTBE reactive distillation, we have applied the unsteady state mass and component equations as our test function for GSA algorithm. The input parameters with their values are given in Table I. We have also used universal bench mark unimodel test functions as proposed by [16] which are given in Table II.

TABLE I: PROCESS PARAMETER OF MTBE RD
\begin{tabular}{|l|l|l|}
\hline $\begin{array}{l}\text { Condition/ } \\
\text { Parameters }\end{array}$ & Methanol & Isobutylene \\
\hline Feed Flow $(\mathrm{mol} / \mathrm{s})$ & 205 & 550 \\
\hline Temperature $\left({ }^{0} \mathrm{~K}\right)$ & 320 & 350 \\
\hline Pressure $($ bar) & 11 & 11 \\
\hline Feed Stage & 4 & 11 \\
\hline No of Stages & 17 \\
\hline Reflux Ratio & 7.1 \\
\hline \% Conversion & 99.9 \\
\hline Reflux Flow $(\mathrm{kmol} / \mathrm{s})$ & 2.5 \\
\hline Distillate Flow $(\mathrm{mol} / \mathrm{s})$ & 28 \\
\hline $\begin{array}{l}\text { Bottom Flow } \\
\text { (mol/s) }\end{array}$ & 17 \\
\hline
\end{tabular}

TABLE II: UNIMODEL BENCHMARK TEST FUNCTIONS

\begin{tabular}{|l|c|}
\hline Test Function & $\mathrm{S}$ \\
\hline$F_{1}(X)=\sum_{i=j}^{n} x_{1}^{2}$ & {$[-100,100]^{n}$} \\
\hline$F_{2}(X)=\sum_{i=j}^{n}\left|x_{i}\right|+\prod_{i=j}^{n}\left|x_{1}\right|$ & {$[-10,10]^{n}$} \\
\hline$F_{3}(X)=\sum_{i=j}^{n}\left(\sum_{j-i}^{i} x_{j}\right)^{2}$ & {$[-100,100]^{n}$} \\
\hline$F_{4}(X)=\max _{i}\left\{\left|x_{i}\right|, 1 \leq i \leq n\right\}$ & {$[-100,100]^{n}$} \\
\hline$F_{5}(X)=\sum_{i=j}^{n-1}\left[100\left(x_{i+j}-x_{i}^{2}\right)^{2}+\right.$ & \\
$\left.\left(x_{i}-1\right)^{2}\right]$ & {$[-100,100]^{n}$} \\
\hline$F_{6}(X)=\sum_{i=j}^{n}\left(\left|x_{1}+0.5\right|\right)^{2}$ & {$[-1.28,1.28]^{n}$} \\
\hline$F_{7}(X)=\sum_{i=j}^{n} i x_{i}^{4}+$ random $[0,1]$ & \\
\hline
\end{tabular}

\section{RESULTS AND DISCUSSION}

In this case, GSA implementation is done using MATLAB. In case of MTBE reactive distillation, both the feed flow rates on reactive zone as well as vapor and liquid flow rates within the column are chosen as active masses along with temperature of the column. The composition and Temperature profile of MTBE Reactive Distillation is shown in Fig. 3 and Fig. 4 respectively. As can be seen in Fig. 3 the change is composition of MTBE is increasing continuously from tray no 1 to 15 and achieved optimized mol fraction of approx 0.8 .

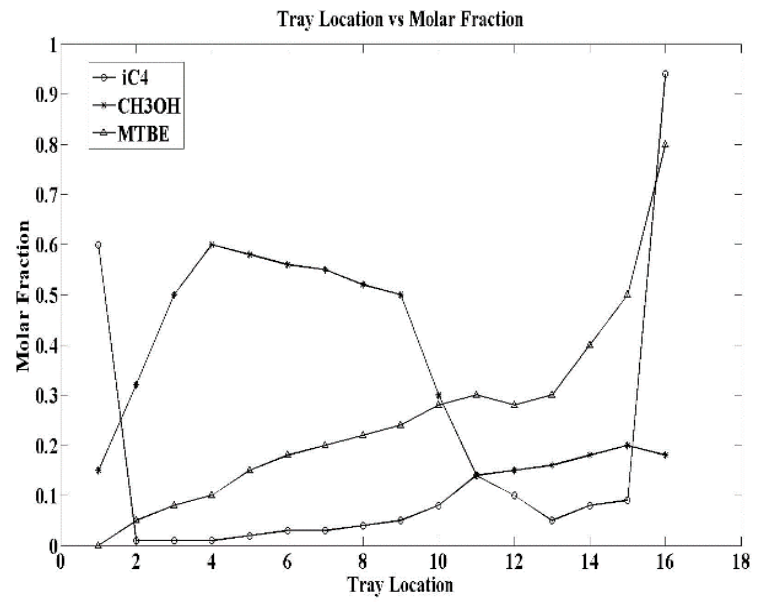

Fig. 3. Composition vs tray location of MTBE reactive distillation. 


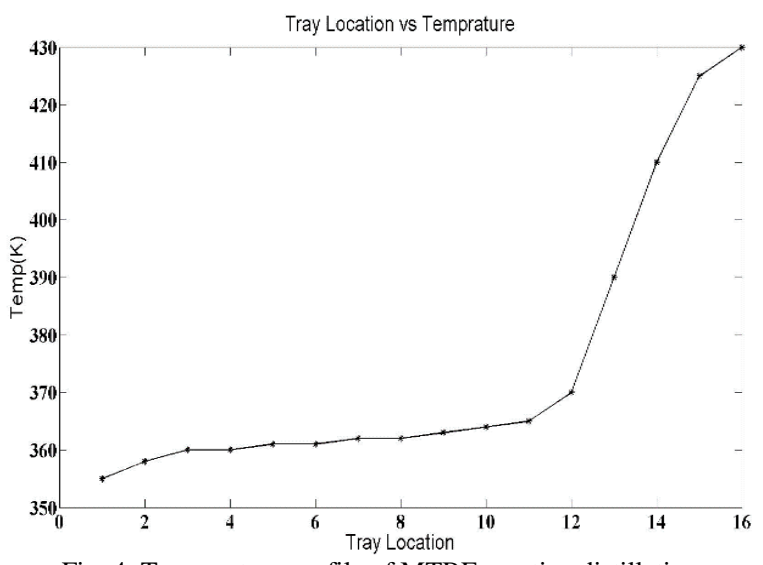

Fig. 4. Temperature profile of MTBE reactive distillation.

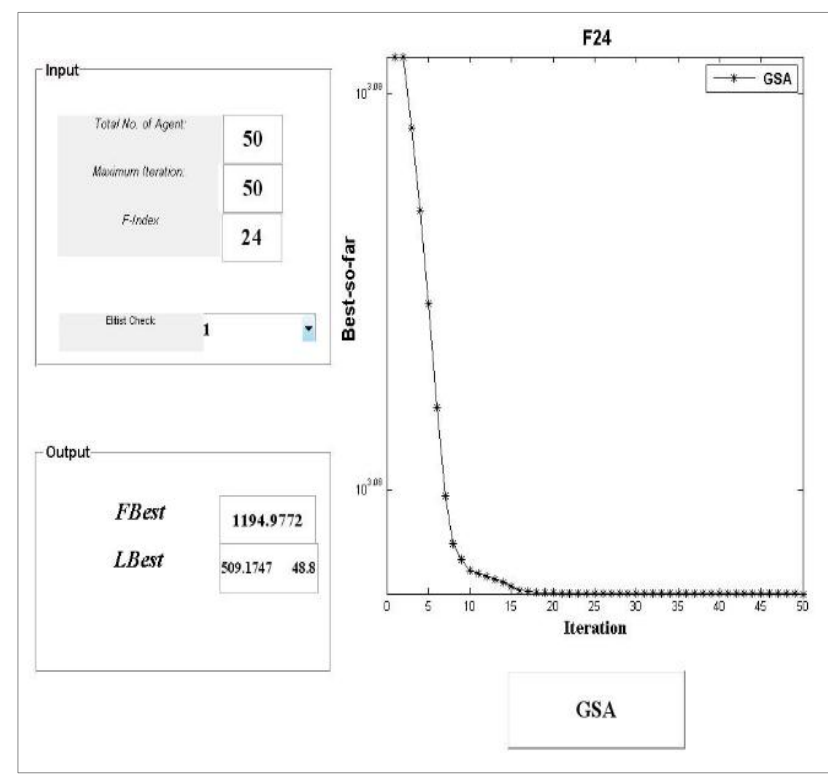

Fig. 5. Performance of MTBE RD by GSA using selected fitness function with 50 iteration.

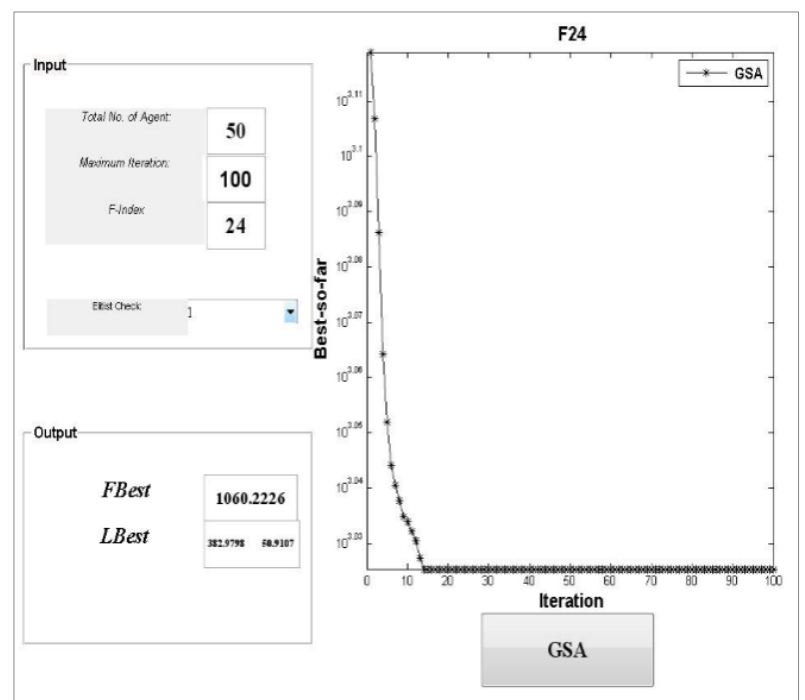

Fig. 6. Performance of MTBE RD by GSA using selected fitness function and 100 iterations.

As it can see in the Fig. 4 the temperature is linear from tray 1 to tray 11 then it increases suddenly between trays 11 to 15 . We can clearly observe in the Fig. 5, Fig. 6 and Fig. 7 shown below, which are obtained using GSA algorithm and using selected test function gives better results. It has been found that after increasing the number of iterations more than 100, no further increase in optimization and it goes straight throughout, therefore we have run our GSA using 50 and 100 iterations to observe the behavior of FBest. We have also implemented GSA algorithm for the benchmark unimodal function F3.

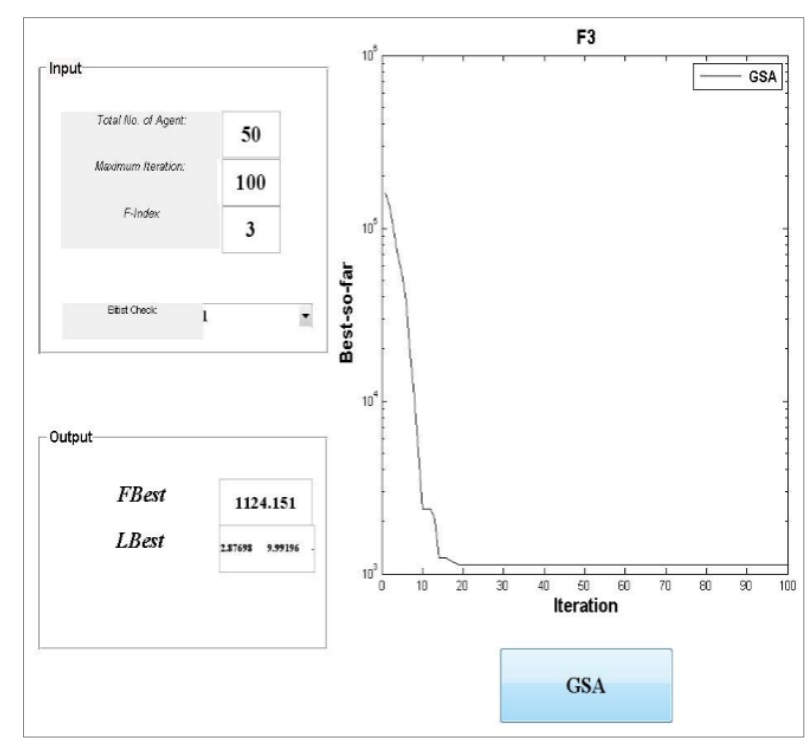

Fig. 7. Performance of MTBE RD by GSA using unimodel test function F3and 100 iterations.

\section{CONCLUSION}

The heuristic optimization algorithm such as GSA, developed recently, is implemented in our system. The system chosen is MTBE reactive distillation. We have applied feed flow rates as our test function along with universal benchmark unimodel test function to obtain optimized process. The GSA code is generated in MATLAB. The performance of GSA has achieved good results regarding the solution quality and success rate in finding optimal solution.

\section{ACKNOWLEDGEMENT}

The author acknowledges the financial assistance provided by All India Council of Technical Education (AICTE), New Delhi under Research Promotion Scheme (RPS) in 2012.

\section{NOTATIONS}

$F \quad$ Feed flow rate

$D \quad$ Distillate flow rate

$B$ Bottoms flow rate

$F_{j} \quad$ Feed Flow Rates at $j^{\text {th }}$ tray

$L_{j-1} \quad$ Liquid flow rate at $j-1^{\text {th }}$ tray

$V_{j+1} \quad$ Vapor flow rate at $j+1^{\text {th }}$ tray

$L_{j} \quad$ Liquid flow rate at $j^{\text {th }}$ tray

$V_{j} \quad$ Vapor flow rate at $j^{\text {th }}$ tray

$H_{j} \quad$ The molar quantity (or volumetric quantity) of liquid mixture that remains or held at certain level on stage $j$ (liquid holdup)

$R_{i} \quad$ total numbers of moles generated or disappear through reaction on stage $j$

$r_{i, r} \quad$ rate of reaction $r$ on stage $j(\mathrm{~mol} / \mathrm{s})$

$z_{j, i,} \quad$ mole fractions of component $i$ of feed flow

$x_{j, i}, \quad$ mol fraction of component $i$ in liquid flow $L_{j}(\mathrm{~mol})$ 
on stage $j$

$y_{j, i} \quad$ mol fraction of component $i$ in vapor flow $v_{j}(\mathrm{~mol})$ on stage $j$

$v_{r, I}$ stoichiometric coefficient of component $i$ for reaction $r$

\section{Subscripts:}

$j \quad$ stage number,

$i \quad$ component number,

$r \quad$ specific reaction number.

$\delta_{j}(0$ or 1$)$ reaction occurrence on stage $j$ (When reaction occurs on stage $j, \delta_{j}$ is set to unity but when there is no reaction; $\delta_{j}$ is set to zero value).

\section{REFERENCES}

[1] M. Z. Mohammed, "Mathematical modeling and simulation for production of MTBE by reactive distillation," Ph.D. dissertation, University of Technology, Saudi Arabia, 2009.

[2] A. K. Jana, K. J. Prakash, and D. S. Patle, "Neuro-estimator based GMC control of batch reactive distillation," ISA Transactions, vol. 50, pp. 357-363, 2011.

[3] Yu-Chu Tian, F. Zhao, B. H. Bisowarno, and M. O. Tade, "Pattern based predictive control for ETBE reactive distillation," Journal of Process Control, vol. 13, issue 1, pp. 57-67, 2001.

[4] E. Zondervan, M. Shah, and A. B. Haan, "Optimal design of reactive distillation column," Chemical Engineering Transactions, vol. 24, pp. 295-300, 2011.

[5] V. Amte, S. H. Nistala, R. K. Malik, and S. Mahajani, "Optimization based conceptual design of reactive distillation for selectivity engineering," Computers and Chemical Engineering, vol. 48, pp. 209-217, 2013

[6] V. Bansal, V. Sakizlis, R. Ross, J. D. Perkins, and E. N. Pistikopoulos, "New algorithm for mixed-integer dynamic optimization," Computers and chemical engineering, vol. 27, pp. 647-668, 2003.

[7] E. Rashedi, H. N. Pour, and S. Saryazdi, "GSA: A gravitational search algorithm," Information Sciences, vol. 179, pp. 2232-2248, 2009.

[8] E. Rashedi, "Gravitational Search Algorithm," M.Sc. thesis, Shahid Bahonar University of Kerman, Kerman, Iran, 2007.

[9] T. Eldos and R. A. Qasim, "On the performance of gravitational search algorithm," International Journal of Advanced Computer Science and Applications (IJACSA), vol. 4, no. 8, pp. 74-78, 2013.

[10] M. N. Murat, A. R. Mohamed, and S. Bhatia, "Modeling of a Reactive Distillation Column: Methyl Tertiary Butyl Ether (MTBE) Simulation Studies," IIUM Engineering Journal, vol. 4, no. 2, pp. 1330, 2003.

[11] D. B. Leineweber, "Efficient reduced SQP methods for the optimization of chemical processes described by large sparse DAE models," Ph.D. dissertation, University of Heidelburg, 1998.

[12] J. Kennedy and R. C. Eberhart, "Particle swarm optimization," in Proc. IEEE International Conference on Neural Networks, vol. 4 1995, pp. 1942-1948.
[13] D. H. Kim, A. A braham, and J. H. Cho, "A hybrid genetic algorithm and bacterial foraging approach for global optimization," Information Sciences, vol. 177, 3918-3937, 2007.

[14] T. H. Kim, I. Maruta, and T. Sugie, "Robust PID controller tuning based on the constrained particle swarm optimization," Automatica, vol. 44, 1104-1110, 2008.

[15] A. Formato, "Central force optimization: A metaheuristic with applications in applied electromagnetic," Progress in Electromagnetics Research, vol. 77, pp. 425-491, 2007.

[16] X. Yao, Y. Liu, and G. Lin, "Evolutionary programming made faster," IEEE Transactions on Evolutionary Computation, vol. 3, pp. 82-102, July 1999 .

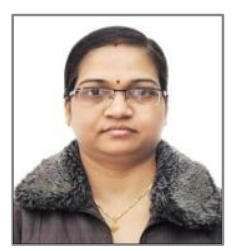

Vandana Sakhre is working as an assistan professor, Department of Chemical Engineering, Madhav Institute of Technology \& Science (MITS), Gwalior, India, an autonomous Government Aided Institute under state technical university of Madhya Pradesh, India, since 2008. She has pursued her bachelor degree in chemical engineering from NIT, Raipur, and M.Tech from Indian School of Mines (ISM), Dhanbad, India. She has 10 years of teaching experience and 5 years of experience in R\&D and industry. Presently she is doing Ph.D. on "Development of Soft Sensors for Reactive Distillation System".

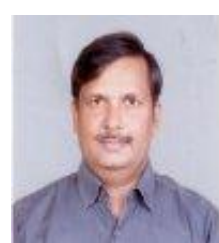

Sanjeev Jain was born in year 1967. He did his bachelors from SATI, Vidisha, M.Tech from IIT Delhi and Ph.D from Barkatullah University, Bhopal (MP), India, in year 2000. Presently he assumed the charge of director, Madhav Institute of Technology \& Science (MITS), Gwalior, India.

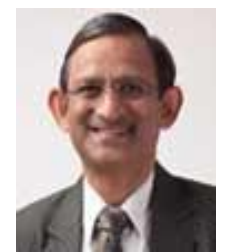

V. S. Sapkal received his bachelor in chemical engineering, M.Tech. \& Ph.D. in chemical engineering from I.I.T., Powai, Mumbai, 1987. Dr. V.S. Sapkal, assumed the charge of vicechancellor (VC), Rashtrasant Tukadoji Maharaj (RTM), Nagpur University, Nagpur, India with effect from December 2010. He was a professor and the head of Department of Chemical Engineering, Amravati University, Ameravati for 15 years. $\mathrm{He}$ worked as expert for industrial health and safety, pollution control board, non-conventional energy sources, etc.

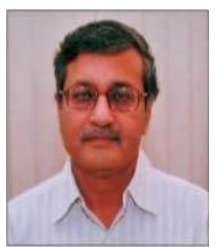

D. P. Agrawal was born on $2^{\text {nd }}$ October, 1949. After graduating in engineering in 1970 from AMU Aligarh, he obtained his Ph.D. from IIT, Delhi, India. Prof. D. P. Agrawal assumed the charge of chairman of the Union Public Service Commission with effect from August 16, 2008, New Delhi, India. 